\title{
Inappropriate elevation of bispectral index values in robot assisted thyroidectomy with electromyographic endotracheal tube
}

\section{-A case report-}

\author{
Mae-Hwa Kang, Chi-Bum In, Man-ho Kim, Kyoung-Ji Lim, Eun Young Park, Hyo Min Lee, and \\ Soo Kyung Lee
}

Department of Anesthesiology and Pain Medicine, College of Medicine, Hallym University, Anyang, Korea

We report a case of an erroneously elevated bispectral index (BIS) during robot assisted thyroidectomy using an electromyographic endotracheal tube (EMG tube), which is safe and useful for laryngeal electromyographic monitoring. Ten minutes after start of the operation, a sudden increase of BIS value up to 98 was noticed. The BIS values were not decreased to $<65$ with supplemental anesthetics. The anesthetic method was changed from total intravenous anesthesia to balanced anesthesia. The BIS sensor and monitor were changed and other models were used. These interventions did not alter BIS values. BIS levels remained between 60 and 70 throughout the main procedure and intermittently increased to the mid-90s without any trace of poor signal quality. At the end of the surgery, the BIS values returned to normal range. The patient did not complain of intraoperative recall. Knowledge of potential interference from the use of an EMG tube must be considered when interpreting BIS. (Korean J Anesthesiol 2011; 61:511-514)

Key Words: Bispectral index, Electromyographic endotracheal tube, Laryngeal nerve monitoring, Thyroid surgery.

Received: June 30, 2011. Revised: 1st, July 27, 2011; 2nd, August 9, 2011. Accepted: August 25, 2011.

Corresponding author: Mae-Hwa Kang, M.D., Ph.D., Department of Anesthesiology and Pain Medicine, Hallym University Sacred Heart Hospital, College of Medicine, Hallym University, 896, Pyeongchon-dong, Dongan-gu, Anyang 431-070, Korea. Tel: 82-31-380-5959, Fax: 82-31385-3244, E-mail: drberry@hanmail.net

(C)This is an open-access article distributed under the terms of the Creative Commons Attribution Non-Commercial License (http:// creativecommons.org/licenses/by-nc/3.0/), which permits unrestricted non-commercial use, distribution, and reproduction in any medium, provided the original work is properly cited. 
Depth of anesthesia is commonly monitored by Bispectral index (BIS) analysis, which is a non invasive technology using an electroencephalogram (EEG) derivative collected from a forehead sensor. BIS has been accepted as an accurate measure of the hypnotic effect of anesthetics and sedative drugs [1]. It is a dimensionless number that varies from 0 to 100 , with 0 indicating an isoelectric EEG and values near 100 associated with an awake state. BIS values between 40 and 60 are associated with a low probability of consciousness and reflect sufficient anesthesia [2]. However, the concurrent use of a variety of medical devices can lead to inaccuracies in its output.

The electromyographic endotracheal tube (EMG tube) was designed to measure integrity of the vocal cord structures during thyroidectomy, and has already been accepted as a safe and useful device [3]. NIM-Response ${ }^{\circledR}$ Nerve Integrity Monitoring System (Medtronic, Jacksonville, FL, USA) is the commonly used device for identifying the recurrent laryngeal nerve.

In this report, we present a case of falsely increased BIS values due to interference from laryngeal electromyography monitoring with a EMG tube used in a robot assisted total thyroidectomy. Paradoxical high BIS values led to unnecessary deep anesthesia with subsequent prolonged emergence.

\section{Case Report}

A healthy 33-year-old female patient (ASA class I) underwent a robot assisted total thyroidectomy and lymph node dissection. She had no significant medical history. Routine preoperative investigations were within normal limits.

After routine monitoring, general anesthesia was initiated with continuous infusion of propofol and remifentanil using a targeted controlled infusion system (Orchestra, Fresinius vial, France), and endotracheal intubation was facilitated by rocuronium (50 mg). The trachea was easily intubated using a Medtronic Xomed Nerve Integrity Monitor (NIM ${ }^{\circledR}$ ) EMG endotracheal tube (Jacksonville, FL, USA). Correct positioning of the wire electrode was verified visually. The optimal depth was defined during subsequent successful intraoperative neuromonitoring. A BIS Quarto sensor (Aspect Medical Systems, Newton, MA, USA) was mounted on the forehead according to the manufacturer's guidelines and connected to the BISVista monitor (Aspect Medical Systems, Newton, MA, USA). Anesthesia was maintained with medical air, oxygen, and intravenous infusion of propofol and remifentanil. Propofol infusion was titrated to maintain a BIS index of 40-60, and remifentanil infusion was titrated to maintain heart rate and arterial blood pressure within $20 \%$ compared with baseline values. Additional muscle relaxant was not used to monitor integrity of nerves throughout the entire period of surgery.
Ten minutes after incision, a sudden increase of BIS a value of 94 was noticed. The target concentrations of propofol and remifentanil were rapidly increased following intravenous injection of midazolam ( $2 \mathrm{mg}$ ). At the time, the vital signs were not changed, suggesting a sufficient depth of anesthesia. The BIS value was maintained between 65 and 75 for 20 minutes and then suddenly increased to 93 . A midazolam supplement ( $2 \mathrm{mg}$ ) was administered. The nociceptive stimuli might be unchanged during the surgical procedure. All infusions were found to be performed satisfactorily, and there were no signs of patient movement or arousal. The propofol and remifentanil infusions were sequentially increased to $7.0 \mu \mathrm{g} / \mathrm{ml}$ and $6.0 \mathrm{ng} /$ $\mathrm{ml}$ (effect site concentrations). After 20 minutes a $5 \mathrm{mg}$ bolus of IV ephedrine was given because arterial blood pressure was $74 / 58 \mathrm{mmHg}$ and heart rate was 56/min. Considering a small possibility of intravenous line dysfunction, propofol infusion was stopped and sevoflurane was started. Though sevoflurane was gradually increased to an end-expiratory concentration of 3.5 vol\%, the BIS value did not decrease to $<65$. Twentyfive minutes later, sevoflurane was discontinued and total intravenous anesthesia (TIVA) was restarted to infuse via another intravenous route. BIS values stayed in the range of 65-70 after the attempt. The BIS Quatro sensor replacement could not significantly change the BIS values. A similar model of the BIS-Vista monitor was applied to verify any monitoring failure. This intervention did not alter BIS values. Then, the BIS values increased to the mid-90s, and an additional $2 \mathrm{mg}$ dose of midazolam was administered. We concluded that the BIS values

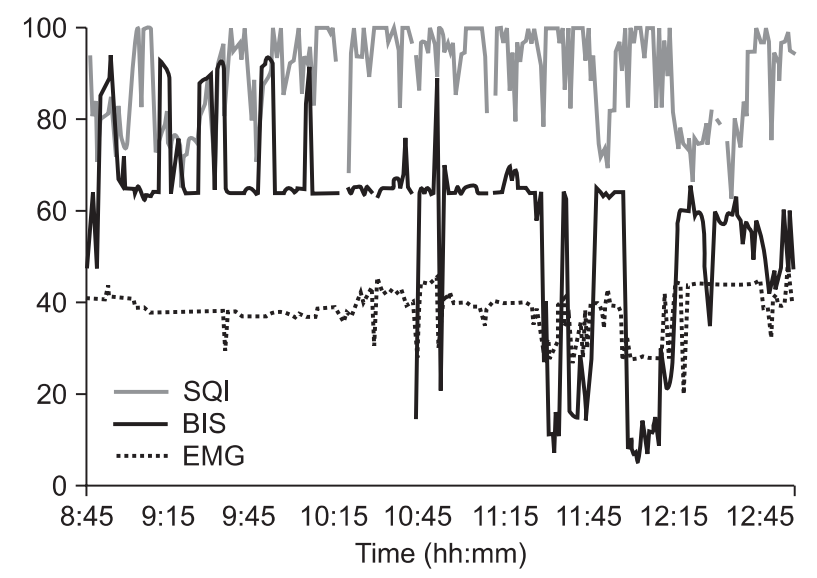

Fig. 1. Trends of bispectral index (BIS), signal quality index (SQI) and electromyography (EMG). The BIS values were in the range of 6070 throughout 3 hours of surgery and intermittently increased to the mid-90s. SQI and EMG did not show poor signal quality during the period indicating high BIS levels. After the end of the main procedure (11:30 A.M.) when laryngeal neuromonitoring was ceased, the BIS declined to a value $<10$. BIS value was maintained within a normal range at the appropriate level of anesthesia during the remaining surgery. 
were inconsistent with sedation level. The settings of propofol and remifentanil were adapted according to clinical signs of the patient during the remaining operating time.

At the end of the main procedure, the BIS declined to a value $<10$. Propofol and remifentanil were rapidly titrated back to a BIS value of 50 . The remainder of the operation proceeded uneventfully and BIS value was maintained within an ideal range at the appropriate level of anesthesia. During the surgery, the EMG graph did not indicate any significant activity $(\sim 40$ $\mathrm{dB}$ ) and the signal quality index (SQI) showed good to excellent signal quality on any device $(>50 \%$, Fig. 1). Both propofol and remifentanil were discontinued at the end of surgery, and the patient was allowed to recover over a 20-minute span. No clear recall or elicit memory during operation was observed when asked once immediately, and then 3 days after anesthesia. The patient was discharged 3 days later.

\section{Discussion}

Recurrent laryngeal nerve injury is one of most feared complications of thyroidectomy. There have been many methods used for intraoperative neuromonitoring to identify recurrent laryngeal nerve. In recent years, wire-reinforced EMG tubes equipped with surface electrodes have gained popularity due to their essential advantages including easy placement, noninvasiveness, and ability to sample larger areas of evoked muscle action potentials [4]. The $\mathrm{NIM}^{\circledR}$ monitor tracks EMG activity continuously, and enables direct stimulation of an exposed nerve and subsequent recording of muscle activity.

BIS monitoring has been proven to be a useful procedure for measuring depth of sedation. However, several cases of BIS values not coinciding with a clinically judged sedative-hypnotic state have been reported. Such reports are as follows: cardiac pacing device [5], warming devices [6], endoscopic shaver device [7], and electromagnetic system [8]. Sloan formerly reported the interference of BIS monitoring with the EMG tube [9]. Our case differs from previous one in that we changed the anesthetics from intravenous agents to a volatile agent, though the manipulation could not alter the BIS values. When measuring EEG in the operating room, it is important to assess the quality of the EEG signal. There are many problems with signal bias due to electrical interference from other equipment used in the operating room. The EMG and SQI values are therefore necessarily recorded to validate the BIS.

Elevated EMG activity and neuromuscular blocking drugs (NMBD) could significantly influence BIS monitoring. EMG activity represents artifacts superimposed on the frequency band of EEG signals used by the algorithm of the monitor to determine BIS values [10]. The administration of NMBD would decrease the BIS value by alleviating the EMG contamination and revealing the true calculated BIS. However, Dahaba et al. [11] and Grief et al. [12] demonstrated that BIS was not altered by neuromuscular block levels. The debate regarding whether or not NMBD affects the value given by sedation depth monitors continues. In our case, the absence of muscle relaxant might cause inaccurate BIS values due to EMG activity. We could not attempt to administer a NMBD due to intraoperative neuromonitoring. However, judging from the phenomenon that the BIS values decreased after cessation of the main procedure, presumed to coincide with discontinuance of EMG stimulation, the EMG interference might be the cause of the erroneous BIS finding. In our center, EMG stimulation generally ends with electrophysiologic confirmation of objective preservation of the recurrent laryngeal nerve after completion of thyroid dissection. Although the EMG scores demonstrated similar levels throughout the duration of the surgery, the appearance of high BIS scores were limited to the period of the main procedures.

Subtle artifact signal pollution arising from electric device interference could not be identified by the signal quality bar nor displayed as artifacts. In this case, the BIS reading was falsely increased despite optimal signal quality indication, and no indication of EMG activity. Recurrent laryngeal nerve stimulation might cause signal interference, simulating EEG waves found in superficial anesthesia or during alertness, which were not identified by the monitor as interferences.

Other factors causing inaccurate BIS values such as nitrous oxide administration, use of electrocautery, and forced air warming device could be excluded. Anesthesia was maintained without nitrous oxide. Electrocautery was used throughout the operation, even in the period indicating appropriate BIS levels before and after the main procedure. Forced air warming devices were not used for this patient.

Although the BIS algorithm has been modified several times to improve its performance and decrease the interference of artifacts, BIS values can still be influenced by different clinical situations that were not included during its development [1]. The BIS Quatro Sensor has a new electrode which offers enhanced artifact detection and improved resistance to interference from noise sources, such as high frequency/electromyography conditions. The BIS-Vista monitor uses a new proprietary algorithm. In our case, the latest model of the BIS system produced inaccurate high values due to interference arising from an extraneous source, as previously described in the prior models [5-9].

In conclusion, we present a case where electrical interference with the EMG tube was misinterpreted as EEG activity by a BISVista monitor. This misinterpretation was displayed as a high BIS value, with optimal signal quality and no significant EMG activity. 


\section{References}

1. Dahaba AA. Different conditions that could result in the bispectral index indicating an incorrect hypnotic state. Anesth Analg 2005; 101: 765-73.

2. Johansen JW. Update on bispectral index monitoring. Best Pract Res Clin Anaesthesiol 2006; 20: 81-99.

3. Eisele DW. Intraoperative electrophysiologic monitoring of the recurrent laryngeal nerve. Laryngoscope 1996; 106: 443-9.

4. Ito H, Sobue K, So M, Hirate H, Sugiura T, Azami T, et al. Intraoperative monitoring of vagal nerve activity with wire electrodes. Acta Anaesthesiol Scand 2006; 50: 1304-5.

5. Gallagher JD. Pacer-induced artifact in the bispectral index during cardiac surgery. Anesthesiology 1999; 90: 636.

6. Guignard B, Chauvin M. Bispectral index increases and decreases are not always signs of inadequate anesthesia. Anesthesiology 2000; 92: 903.

7. Hemmerling TM, Migneault B. Falsely increased bispectral index during endoscopic shoulder surgery attributed to interferences with the endoscopic shaver device. Anesth Analg 2002; 95: 1678-9.

8. Hemmerling TM, Desrosiers M. Interference of electromagnetic operating systems in otorhinolaryngology surgery with bispectral index monitoring. Anesth Analg 2003; 96: 1698-9.

9. Sloan PA. Interference of bispectral index monitoring with intraoperative use of the electromyograph endotracheal tube. Can J Anaesth 2007; 54: 1028-9.

10. Sleigh JW, Steyn-Ross DA, Steyn-Ross ML, Williams ML, Smith P. Comparison of changes in electroencephalographic measures during induction of general anaesthesia: influence of the gamma frequency band and electromyogram signal. Br J Anaesth 2001; 86: 50-8.

11. Dahaba AA, Mattweber M, Fuchs A, Zenz W, Rehak PH, List WF et al. The effect of different stages of neuromuscular block on the bispectral index and the bispectral index-xp under remifentanil/ propofol anesthesia. Anesth Analg 2004; 99: 781-7.

12. Greif R, Greenwald S, Schweitzer E, Laciny S, Rajek A, Caldwell $\mathrm{JE}$, et al. Muscle relaxation does not alter hypnotic level during propofol anesthesia. Anesth Analg 2002; 94: 604-8. 